\title{
NANOG expression correlates with differentiation, metastasis and resistance to preoperative adjuvant therapy in oral squamous cell carcinoma
}

\author{
MASAHIRO WATANABE ${ }^{1-3}$, YUICHI OHNISHI ${ }^{2,3}$, HIROSHI INOUE $^{3,4}$, \\ MASAHIRO WATO $^{5}$, AKIO TANAKA ${ }^{5}, \mathrm{KENJI} \mathrm{KAKUDO}^{3}$ and MASAMI NOZAKI ${ }^{2}$ \\ ${ }^{1}$ Graduate School of Dentistry, Osaka Dental University, Hirakata, Osaka 573-1121; ${ }^{2}$ Department of Cell Biology, \\ Research Institute for Microbial Diseases, Osaka University, Suita, Osaka 565-0871; \\ ${ }^{3}$ Second Department of Oral and Maxillofacial Surgery, Osaka Dental University, Hirakata, Osaka 573-1121; \\ ${ }^{4}$ Department of Dentistry and Maxillofacial Surgery, Osaka Red Cross Hospital, Osaka 543-8555; \\ ${ }^{5}$ Oral Pathology, Osaka Dental University, Hirakata, Osaka 573-1121, Japan
}

Received April 17, 2013; Accepted October 28, 2013

DOI: $10.3892 / \mathrm{ol} .2013 .1690$

\begin{abstract}
NANOG protein, a transcription factor expressed in embryonic stem cells, is overexpressed in tumor development. Although studies investigating the function of NANOG in cancer have shown that it plays several roles, such as in cell proliferation, invasion and metastasis, the overall function of NANOG in cancer cells has remained elusive. In the present study, NANOG expression in oral squamous cell carcinoma (OSCC) was examined to determine its potential clinical significance. The expression of NANOG protein was assessed in 60 patients with OSCC by immunohistochemistry, and its correlation with clinicopathological factors and metastasis was evaluated. NANOG protein levels in human OSCC cell lines were determined by western blotting and immunofluorescence staining. NANOG protein expression was identified in 52 cases $(86.7 \%)$ and expression levels were higher in primary foci of poorly differentiated OSCC than in those of well-differentiated OSCC, indicating that NANOG expression is associated with OSCC differentiation. Regardless of the differentiation levels of primary foci, NANOG expression levels in metastatic foci were extremely high. In addition, NANOG expression in metastatic foci was maintained at high levels following preoperative adjuvant therapy. Furthermore, NANOG protein was detected at an identical level in human OSCC cell lines. These data indicate that NANOG-expressing OSCC cells tend to metastasize and that metastatic tumors expressing NANOG may be resistant
\end{abstract}

Correspondence to: Dr Masami Nozaki, Department of Cell Biology, Research Institute for Microbial Diseases, Osaka University, 3-1 Yamadaoka, Suita, Osaka 565-0871, Japan

E-mail:mnozaki@biken.osaka-u.ac.jp

Key words: oral squamous cell carcinoma, NANOG, differentiation, metastasis, preoperative adjuvant therapy to preoperative adjuvant therapy, including chemoradiation. Thus, assessment of NANOG expression may assist the strategy for treatment of OSCC metastasis.

\section{Introduction}

Head and neck squamous cell carcinoma, which includes oral squamous cell carcinoma (OSCC), is the sixth most prevalent malignancy worldwide $(1,2)$. Due to the poor prognosis of OSCC, the overall five-year survival rate of patients following surgical resection has not improved markedly during the past three decades (3).

The transcription factor NANOG is critical for the regulation of cell fate in the inner cell mass during embryonic development and pluripotency of embryonic stem cells (4-7). Overexpression of NANOG protein has been previously found in a variety of tumors, including breast cancer (8), colorectal cancer $(9,10)$, gastric carcinoma (11) and OSCC $(12,13)$. Previous studies report variable NANOG expression, from undetectable to extremely high levels, in OSCC samples. Furthermore, NANOG expression may be associated with patient survival. Elevated NANOG expression has been found to be associated with a poor prognosis, advanced stage and medially-to-poorly differentiated OSCC $(14,15)$. Based on these observations, NANOG may be a useful prognosis factor. However, the correlation among NANOG expression, differentiation and metastasis in OSCC remains unclear.

In this study, NANOG expression in OSCC specimens was examined by immunohistochemistry. Furthermore, the association between NANOG expression and differentiation, metastatic potency and resistance of OSCC to preoperative adjuvant therapy was evaluated.

\section{Materials and methods}

Patients. Between 1997 and 2011, 60 patients with operable oral cancer underwent surgery at the Department of Oral and Maxillofacial Surgery (Osaka Dental University Hospital 
Table I. Clinicopathological factors in 60 patients with OSCC.

\begin{tabular}{|c|c|c|}
\hline Variable & $\begin{array}{c}\text { Well- } \\
\text { differentiated }\end{array}$ & $\begin{array}{c}\text { Poorly } \\
\text { differentiated }\end{array}$ \\
\hline \multicolumn{3}{|l|}{ Gender, $\mathrm{n}$} \\
\hline Male & 18 & 18 \\
\hline Female & 19 & 5 \\
\hline \multicolumn{3}{|l|}{ Age, years } \\
\hline Mean & 65.6 & 63.5 \\
\hline Range & $18-84$ & $47-81$ \\
\hline \multicolumn{3}{|l|}{ Region, $\mathrm{n}$} \\
\hline Tongue & 20 & 5 \\
\hline Gingiva & 10 & 11 \\
\hline Floor of oral cavity & 2 & 6 \\
\hline Buccal mucosa & 4 & 1 \\
\hline Palate & 1 & 0 \\
\hline \multicolumn{3}{|l|}{ T status, $\mathrm{n}$} \\
\hline $\mathrm{T} 1$ & 11 & 5 \\
\hline $\mathrm{T} 2$ & 17 & 12 \\
\hline $\mathrm{T} 3$ & 8 & 3 \\
\hline $\mathrm{T} 4$ & 1 & 3 \\
\hline \multicolumn{3}{|l|}{$\mathrm{N}$ status, $\mathrm{n}$} \\
\hline No & 20 & 16 \\
\hline N1 & 6 & 1 \\
\hline $\mathrm{N} 2 \mathrm{a}$ & 0 & 0 \\
\hline $\mathrm{N} 2 \mathrm{~b}$ & 11 & 6 \\
\hline N3 & 0 & 0 \\
\hline
\end{tabular}

OSCC, oral squamous cell carcinoma.

Table II. Preoperative adjuvant therapy regimen.

\begin{tabular}{lll}
\hline Patient no. & Differentiation level & \multicolumn{1}{c}{ Regimen } \\
\hline 1 & Well-differentiated & PEP + RT \\
2 & Well-differentiated & PEP + CDDP + TS-1 + RT \\
3 & Well-differentiated & TS-1 + RT \\
4 & Poorly differentiated & PEP + RT \\
5 & Poorly differentiated & CDDP + 5-FU \\
6 & Poorly differentiated & TS-1 + RT \\
7 & Poorly differentiated & PEP + RT \\
8 & Poorly differentiated & CDDP + 5-FU + RT \\
9 & Well-differentiated & PEP + RT \\
10 & Well-differentiated & PEP + CDDP + RT \\
11 & Well-differentiated & PEP + CDDP + RT
\end{tabular}

PEP, pepleomycin; RT, radiation therapy; CDDP, cisplatin; TS-1, tegafur + gimeracil + oteracil potassium; 5-FU, 5-fluorouracil.

Hirakata, Japan; Table I). This study follows the tenets of the Declaration of Helsinki and was approved by the ethics committee of Osaka Dental University Hospital (Osaka, Japan). Informed consent was obtained from the patients. None of the primary foci were subjected to preoperative adjuvant therapy and, among 24 metastatic samples, 11 were from patients who underwent preoperative adjuvant therapy. The constituents of the adjuvant therapy are shown in Table II. Tumors were evaluated histologically, based on the International Union Against Cancer classification (16).

Immunohistochemistry. Tissue samples of oral cancers of various stages from patients were fixed in $10 \%$ neutral-buffered formalin solution immediately following resection and were embedded in paraffin. Sections of $4-\mu \mathrm{m}$ thickness were cut and mounted on silane-coated glass slides. The sections were deparaffinized in d-limonene and dehydrated in a graded ethanol series. Antigen retrieval was performed by autoclaving at $121^{\circ} \mathrm{C}$ for $15 \mathrm{~min}$ in Tris-EDTA buffer ( $\mathrm{pH}$ 9.0). Endogenous peroxidase activity was blocked by incubation with $3 \% \mathrm{H}_{2} \mathrm{O}_{2}$ for $10 \mathrm{~min}$ and nonspecific reactions were blocked by incubation with blocking solution (Nacalai Tesque, Inc., Kyoto, Japan) for $10 \mathrm{~min}$. The tissue sections were incubated with goat anti-NANOG polyclonal antibody (1:300; Abnova, Taipei, Taiwan) at room temperature for $1 \mathrm{~h}$. Tissue sections were then incubated with anti-goat $\mathrm{IgG}$ peroxidase-conjugated micropolymer (Vector Laboratories, Burlingame, CA, USA) at room temperature for $30 \mathrm{~min}$ and visualized by incubation with 3,3'-diaminobenzidine tetrahydrochroride liquid system (Dako, Tokyo, Japan) at room temperature for $5 \mathrm{~min}$. The sections were counterstained with hematoxylin and observed by light microscopy (Olympus Corporation, Tokyo, Japan).

Evaluation of slides. NANOG protein immunoreactivity was evaluated by two independent pathologists who had no knowledge of the patient's clinicopathological factors and outcomes. Nuclear expression of NANOG protein was scored semiquantitatively by the combination of intensity (1, weak staining; 2, moderate staining; and 3, strong staining) and the proportion of positively stained tumor cells per 1,000 tumor cells in high-power fields $(1,<25 \% ; 2,25-50 \% ; 3,51-75 \%$; and $4,>75 \%$ ). The sum of the staining intensity and percentage of positive tumor cell scores was graded as follows:,$+ 2-3$; ++ , 4-5; and,$+++ 6-7$. There were no discrepancies between the two pathologists in the overall interpretation of the immunohistochemistry results.

Statistical analysis. Mann-Whitney U tests were performed using the SPSS software (version 13.0; SPSS, Inc., Chicago, IL, USA) to identify statistically significant differences between samples. Data are presented as the mean \pm SD. $\mathrm{P}<0.05$ was considered to indicate a statistically significant difference.

Cell culture. Human SAS, HSC-3 and HSC-4 OSCC cell lines (RIKEN BioResource Center, Ibaraki, Japan) were cultured in DMEM supplemented with $10 \%$ fetal calf serum (both Invitrogen Life Technologies, Carlsbad, CA, USA) at $37^{\circ} \mathrm{C}$ in a humidified atmosphere of $95 \%$ air and $5 \% \mathrm{CO}_{2}$. Cell monolayers were prepared by plating on $10-\mathrm{cm}$ cell culture dishes (Asahi Glass, Tokyo, Japan).

Western blotting. Proteins were resolved in RIPA Buffer [150 mM NaCl, $1.0 \%$ Triton X-100, 0.5\% sodium deoxycholate, 
A

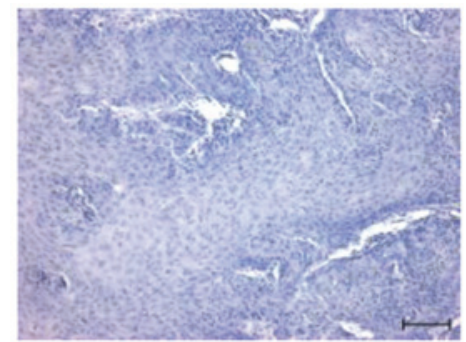

C

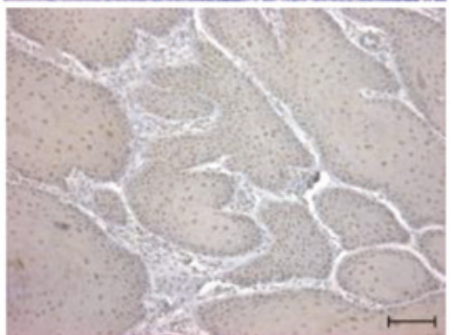

E

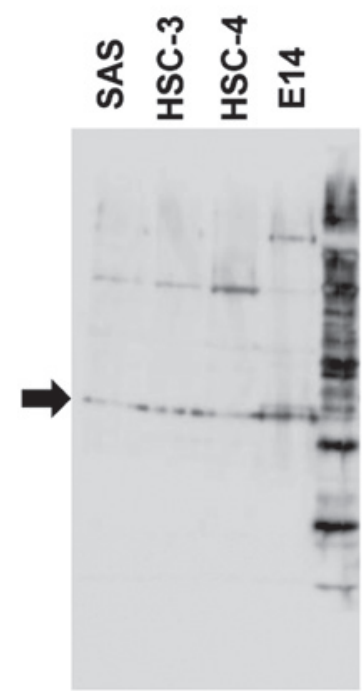

B

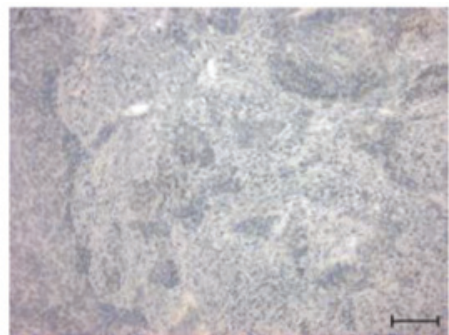

D

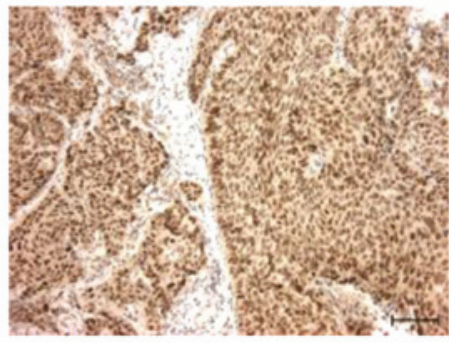

F

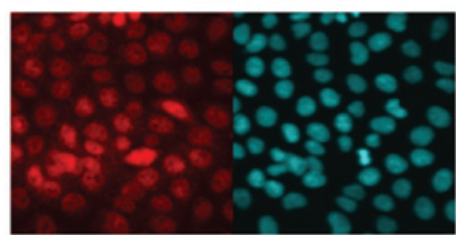

G

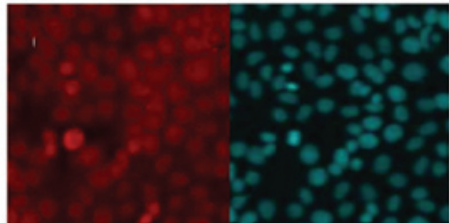

H

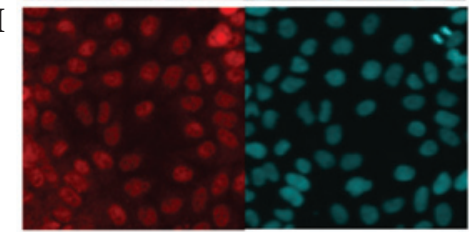

Figure 1. Expression of NANOG protein in OSCC tissues and cell lines. (A) Negative expression of NANOG in OSCC tissues (-). (B) Weak expression of NANOG in OSCC tissues (+). (C) Moderate expression of NANOG in OSCC tissues (++). (D) Strong expression of NANOG in OSCC tissues (+++) (scale bars, $100 \mu \mathrm{m})$. (E) Western blot analysis of NANOG protein expression in SAS, HSC-3 and HSC-4 cells. Nanog protein in mouse embryonic stem cells (E14) was used as a positive control for western blotting (arrow indicates $35 \mathrm{kDa}$ ). Immunocytochemical analysis of NANOG protein expression in (F) SAS, (G) HSC-3 and (H) HSC-4 cells. Right panels show 4',6-diamidino-2-phenylindole staining in the nuclei of the cells. OSCC, oral squamous cell carcinoma.

$0.1 \%$ SDS and $50 \mathrm{mM}$ Tris- $\mathrm{HCl}(\mathrm{pH}$ 8.0)] and separated by $10 \%$ SDS-PAGE. A rabbit anti-NANOG antibody (Abcam, Cambridge, UK) was used as the primary antibody and peroxidase-linked ECL anti-rabbit IgG (GE Healthcare Japan, Tokyo, Japan) was used as the secondary antibody. ECL plus (GE Healthcare Japan) was used as the substrate for western blotting.

Immunofluorescence staining. Cultured cells were fixed with $3.5 \%$ formaldehyde, permeabilized with $0.2 \%$ Triton X-100 and blocked with Image-iT ${ }^{\mathrm{TM}}$ FX Signal Enhancer (Invitrogen Life Technologies). Rabbit anti-NANOG antibody (Abcam) was used as the primary antibody. Next, Alexa Fluor 594-conjugated IgG (Molecular Probes, Eugene, OR, USA) was used as the secondary antibody. Following incubation with the antibodies, SlowFade ${ }^{\circledR}$ Gold antifade reagent with 4',6-diamidino-2-phenylindole (Invitrogen Life Technologies) was added and coverslips were mounted. The specimens were observed using a laser scanning confocal microscope (FV10i-DOC; Olympus Corporation).

\section{Results}

NANOG protein expression in OSCC patients and OSCC cell lines. NANOG protein was clearly stained in the nuclei of cells at various levels in OSCC specimens. Among 60 paraffin-embedded OSCC tissues of primary focus, eight cases (13.3\%) were negative (-), 15 (25\%) showed weak expression (+), $22(36.7 \%)$ showed moderate expression (++) and $15(25 \%)$ showed strong expression (+++). Representative cases of the different NANOG protein expression levels are shown in Fig. 1A-D. To confirm the expression of NANOG in OSCC cell lines, NANOG protein levels were analyzed in SAS, HSC-3 and HSC-4 cells derived from tongue SCCs by western blotting and immunofluorescence staining. NANOG protein was detectable at the same levels in all three cell lines (Fig. 1E-H).

High NANOG protein expression in poorly differentiated OSCC and metastatic foci of OSCC. NANOG protein expression levels were higher in primary foci of poorly differentiated 
Table III. Correlation between NANOG expression and clinicopathological factors in 60 patients with OSCC.

\begin{tabular}{|c|c|c|c|c|c|c|}
\hline \multirow[b]{2}{*}{ Variable } & \multirow[b]{2}{*}{ Negative, $\mathrm{n}$} & \multirow[b]{2}{*}{ Positive, $\mathrm{n}$} & \multicolumn{3}{|c|}{ Expression, $\mathrm{n}$} & \multirow[b]{2}{*}{ P-value } \\
\hline & & & + & ++ & +++ & \\
\hline Total patients & 8 & 52 & 15 & 22 & 15 & \\
\hline \multicolumn{7}{|l|}{ Gender } \\
\hline Male & 4 & 32 & 10 & 8 & 14 & NS \\
\hline Female & 4 & 20 & 5 & 14 & 1 & \\
\hline \multicolumn{7}{|l|}{ Region } \\
\hline Tongue & 0 & 25 & 9 & 12 & 4 & NS \\
\hline Gingiva & 5 & 16 & 4 & 6 & 6 & \\
\hline Floor of oral cavity & 1 & 7 & 1 & 1 & 5 & \\
\hline Buccal mucosa & 2 & 3 & 0 & 3 & 0 & \\
\hline Palate & 0 & 1 & 1 & 0 & 0 & \\
\hline \multicolumn{7}{|l|}{$\mathrm{T}$ status } \\
\hline $\mathrm{T} 1$ & 1 & 15 & 3 & 9 & 3 & NS \\
\hline $\mathrm{T} 2$ & 4 & 25 & 9 & 9 & 7 & \\
\hline T3 & 1 & 10 & 3 & 4 & 3 & \\
\hline $\mathrm{T} 4$ & 2 & 2 & 0 & 0 & 2 & \\
\hline \multicolumn{7}{|l|}{$\mathrm{N}$ status } \\
\hline N1 & 0 & 5 & 0 & 2 & 3 & NS \\
\hline $\mathrm{N} 2 \mathrm{a}$ & 0 & 0 & 0 & 0 & 0 & \\
\hline $\mathrm{N} 2 \mathrm{~b}$ & 0 & 8 & 0 & 1 & 7 & \\
\hline N3 & 0 & 0 & 0 & 0 & 0 & \\
\hline \multicolumn{7}{|l|}{ Primary tumor } \\
\hline Well-differentiated & 4 & 33 & 15 & 18 & 0 & $\mathrm{P}<0.01$ \\
\hline Poorly differentiated & 4 & 19 & 0 & 4 & 15 & \\
\hline \multicolumn{7}{|l|}{ Metastasis } \\
\hline Well-differentiated & 0 & 11 & 0 & 3 & 8 & NS \\
\hline Poorly differentiated & 0 & 2 & 0 & 0 & 2 & \\
\hline Recieved adjuvant therapy & 0 & 11 & 0 & 4 & 7 & NS \\
\hline No adjuvent therapy & 0 & 13 & 0 & 3 & 10 & \\
\hline
\end{tabular}

OSCC, oral squamous cell carcinoma; T, stage of primary tumor; $\mathrm{N}$, stage of lymph node metastasis; +, weak; ++, moderate; +++, strong.

OSCC than in those of well-differentiated OSCC $(\mathrm{P}<0.01$ Table III; Fig. 2A). However, NANOG expression did not correlate with gender, region or $\mathrm{T}$ (stage of primary tumor) and $\mathrm{N}$ (stage of lymph node metastasis) status ( $\mathrm{P}>0.05$; Table III). In well-differentiated OSCC, NANOG expression in metastatic foci was elevated in comparison with its level in primary foci $(\mathrm{P}<0.01$; Table III; Fig. 2B). Representative cases are shown in Fig. 2E and F. However, in metastatic foci, there was no significant association between NANOG expression and differentiation levels ( $>>0.05$; Table III; Fig. 2C). Similarly, among primary and metastatic foci of poorly differentiated OSCC, no significant differences according to NANOG expression were identified ( $\mathrm{P}>0.05$; Table III; Fig. 2D).

High NANOG expression is maintained in metastatic foci with preoperative adjuvant therapy. To investigate the association between NANOG expression and preoperative adjuvant therapy in OSCC, NANOG levels in metastatic lymph nodes were compared between patients who received preoperative adjuvant therapy and those who did not. There was no significant difference between the two groups ( $\mathrm{P}>0.05$; Table III; Fig. 2G). Moreover, OSCC cells (except for those in necrotic tissue) in metastatic lymph nodes subjected to adjuvant therapy expressed NANOG at high levels (Fig. 2H).

\section{Discussion}

Our results show that the nuclei of cancer cells in the majority of OSCC samples (86.7\%) were NANOG-positive. NANOG protein expression levels were higher in poorly differentiated OSCC than in well-differentiated OSCC, and NANOG was detected in all nuclei of OSCC cell lines examined. Furthermore, regardless of preoperative adjuvant therapy, NANOG expression in metastatic foci was extremely high. Although a number of the primary foci $(13.3 \%)$ were negative for NANOG expression, all corresponding metastatic foci expressed high levels of NANOG. 
A

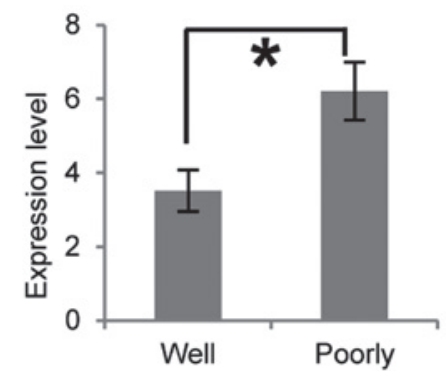

C



$\mathbf{E}$

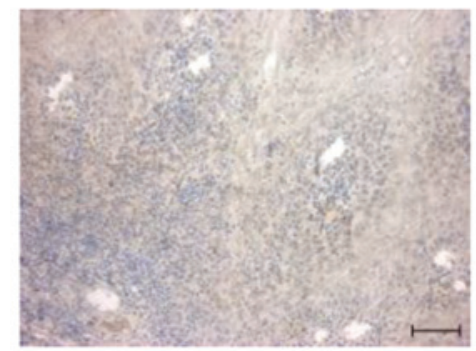

G

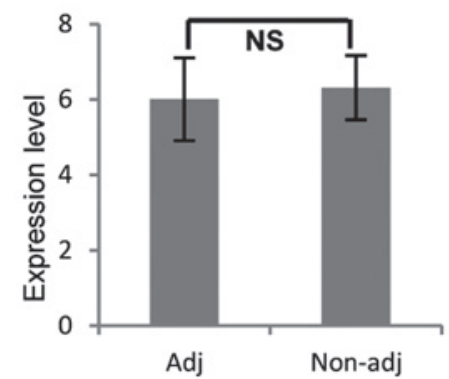

B

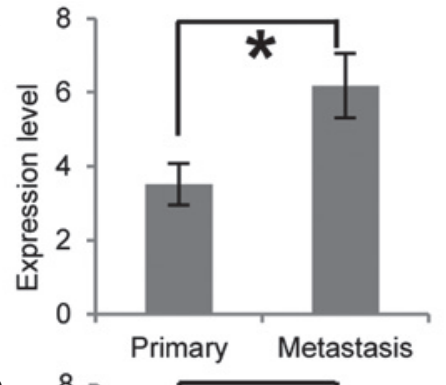

D

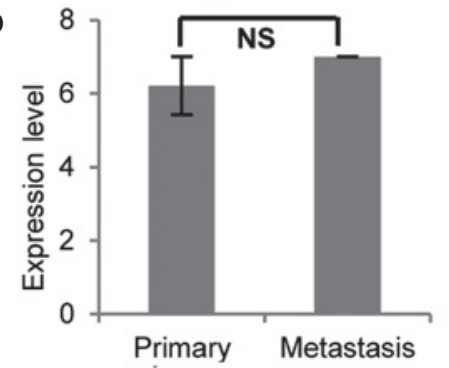

F

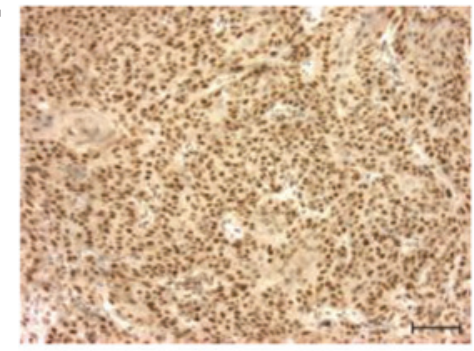

H



Figure 2. High level NANOG expression in tissue samples from metastatic foci in the OSCC patients. (A) Difference in NANOG expression levels between well-differentiated and poorly differentiated primary foci. (B) Difference in NANOG expression levels between primary and metastatic foci of well-differentiated OSCC. (C) Difference in NANOG expression levels between well-differentiated and poorly differentiated metastatic foci. (D) Difference in NANOG expression levels between primary and metastatic foci of poorly differentiated OSCC. (E) Weak (+) NANOG expression in primary focus. (F) Strong (+++) NANOG expression in the metastatic focus. (G) Difference in NANOG expression levels in metastatic foci between patients who received preoperative adjuvant therapy (Adj) and those who did not (Non-adj). (H) NANOG overexpression in a metastatic focus of a patient who received preoperative adjuvant therapy. Asterisk indicates necrotic tissue. Where applicable, data are presented as the mean $\pm \mathrm{SD}\left({ }^{*} \mathrm{P}<0.01\right.$; Mann-Whitney $\mathrm{U}$ test $)$ and scale bars represent $100 \mu \mathrm{m}$. OSCC, oral squamous cell carcinoma; NS, not significant.

Previous studies have shown that almost all tumors are heterogeneous (17-21). Cancer stem cells (CSCs), a small subpopulation of tumor cells, are the main factor in the initiation, growth, metastasis (14) and resistance of chemotherapy of tumors (22), therefore, cancer tends to recur. NANOG protein has been reported to be important in various tumor types, including OSCC (50\% of primary foci and $66.7 \%$ of metastatic foci express NANOG protein) (14), colorectal cancer (20\% of tumors) (10) and gastric carcinoma $(10 \%$ of tumors) (11). In the present study, overexpression of NANOG was detected in poorly differentiated OSCC. Well-differentiated OSCC consists of numerous differentiated cells in the central region of the tumor and the majority undifferentiated tumor cells expressing NANOG exist at the fringe of focus. Immunofluorescence staining showed that OSCC cell lines expressed NANOG protein at the same level. These data indicate that NANOG is expressed not only in CSCs, but also in a large proportion of OSCC cells that are undifferentiated and highly proliferative. Previous studies indicate that NANOG promotes dedifferentiation of p53-deficient mouse astrocytes into brain cancer stem-like cells (23) and blocks differentiation, indicating that, in addition to its importance in CSCs, NANOG plays a significant role in maintaining the non-differentiation or proliferation of OSCC cells. Although the sensitivity of the present immunohistochemistry technique was higher than that in previous studies, specific samples were negative and all were confirmed to express the cell cycle marker Ki-67 (data not shown). These data indicate that 
NANOG is associated with proliferation, independently of the cell cycle, in undifferentiated OSCC cells, including CSCs. In OSCC patients with primary foci in which there was no expression of NANOG, metastatic foci markedly expressed NANOG. As aforementioned, NANOG-negative tumors may contain a limited number of undifferentiated OSCC cells, including CSCs. A previous study showed that high expression of NANOG was associated with metastasis (14). Therefore, in NANOG-negative patients, CSCs expressing NANOG in early stage primary foci metastasize and form the secondary tumor. Thereafter, NANOG-positive undifferentiated cancer cells may be maintained in metastatic foci and disappear from primary foci.

Sentinel lymph node biopsy, which is commonly used to aid breast cancer and melanoma staging, is effective in the diagnosis of OSCC metastasis (24). Immunohistochemistry is required to identify micrometastases and isolated tumor cells (25). The present study indicates that assessment of NANOG protein levels may be useful in sentinel lymph node biopsy.

In the present study, metastatic foci, with or without preoperative adjuvant therapy, showed extremely high expression of NANOG, although necrotic tissues were present within tumors in metastatic lymph nodes subjected to adjuvant therapy. These data indicate that specific tumor cells were necrotized by preoperative adjuvant therapy and that surviving NANOG-positive tumor cells proliferated. A previous study showed that preoperative adjuvant therapy for oral cancer did not significantly improve the survival rate despite the primary local control rate being improved (26). NANOG expression is positively associated with chemoresistance of OSCC $(12,13)$, and CSCs express high levels of NANOG and exhibit high levels of chemoresistance (22). Thus, it is possible that specific tumor cells that did not express NANOG underwent cell death, while undifferentiated tumor cells, including CSCs overexpressing NANOG, survived and continued to proliferate in patients who underwent preoperative adjuvant therapy.

The results of the present study demonstrate that undifferentiated cancer cells overexpressing NANOG are important for metastatic OSCC. Therefore, we hypothesize that targeting NANOG protein may be a useful strategy for the treatment of OSCC metastasis.

\section{References}

1. Chen YJ, Lin SC, Kao T, et al: Genome-wide profiling of oral squamous cell carcinoma. J Pathol 204: 326-332, 2004.

2. Pentenero M, Gandolfo S and Carrozzo M: Importance of tumor thickness and depth of invasion in nodal involvement and prognosis of oral squamous cell carcinoma: a review of the literature. Head Neck 27: 1080-1091, 2005.

3. Myers JN, Elkins T, Roberts D and Byers RM: Squamous cell carcinoma of the tongue in young adults: increasing incidence and factors that predict treatment outcomes. Otolaryngol Head Neck Surg 122: 44-51, 2000.

4. Chambers I, Colby D, Robertson M, et al: Functional expression cloning of Nanog, a pluripotency sustaining factor in embryonic stem cells. Cell 113: 643-655, 2003.
5. Mitsui K, Tokuzawa Y, Itoh H, et al: The homeoprotein Nanog is required for maintenance of pluripotency in mouse epiblast and ES cells. Cell 113: 631-642, 2003.

6. CavaleriF and Schöler HR: Nanog: a new recruit to the embryonic stem cell orchestra. Cell 113: 551-552, 2003.

7. Pan G and Thomson JA: Nanog and transcriptional networks in embryonic stem cell pluripotency. Cell Res 17: 42-49, 2007.

8. Ezeh UI, Turek PJ, Reijo RA and Clark AT: Human embryonic stem cell genes OCT4, NANOG, STELLAR, and GDF3 are expressed in both seminoma and breast carcinoma. Cancer 104: 2255-2265, 2005.

9. Meng HM, Zheng P, Wang XY, et al: Overexpression of nanog predicts tumor progression and poor prognosis in colorectal cancer. Cancer Biol Ther 9: 295-302, 2010.

10. Xu F, Dai C, Zhang R, Zhao Y, Peng S and Jia C: Nanog: a potential biomarker for liver metastasis of colorectal cancer. Dig Dis Sci 57: 2340-2346, 2012.

11. Matsuoka J, Yashiro M, Sakurai K, et al: Role of the stemness factor sox 2 , oct $3 / 4$, and nanog in gastric carcinoma. J Surg Res 174: 130-135, 2012.

12. Tsai LL, Yu CC, Chang YC, Yu CH and Chou MY: Markedly increased Oct4 and Nanog expression correlates with cisplatin resistance in oral squamous cell carcinoma. J Oral Pathol Med 40: 621-628, 2011

13. Bourguignon LY, Earle C, Wong G, Spevak CC and Krueger K: Stem cell marker (Nanog) and Stat-3 signaling promote MicroRNA-21 expression and chemoresistance in hyaluronan/CD44-activated head and neck squamous cell carcinoma cells. Oncogene 31: 149-160, 2012.

14. Chiou SH, Yu CC, Huang CY, et al: Positive correlations of oct-4 and nanog in oral cancer stem-like cells and high-grade oral squamous cell carcinoma. Clin Cancer Res 14: 4085-4095, 2008.

15. Zhang Q, Shi S, Yen Y, Brown J, Ta JQ and Le AD: A subpopulation of CD133(+) cancer stem-like cells characterized in human oral squamous cell carcinoma confer resistance to chemotherapy. Cancer Lett 289: 151-160, 2010.

16. Sobin LH and Wittekind C: International Union Against Cancer TNM classification of malignant tumors. 5th edition. Wiley-Liss Publications, Hoboken, NJ, 1997.

17. Dalerba P, Cho RW and Clarke MF: Cancer Stem Cells: Models and Concepts. Annu Rev Med 58: 267-284, 2007.

18. Clarke MF, Dick JE, Dirks PB, et al: Cancer stem cells - perspectives on current status and future directions: AACR Workshop on cancer stem cells. Cancer Res 66: 9339-9344, 2006.

19. Reya T, Morrison SJ, Clarke MF and Weissman IL: Stem cells, cancer, and cancer stem cell. Nature 414: 105-111, 2001.

20. Costea DE, Tsinkalovsky O, Vintermyr OK, Johannessen AC and Mackenzie IC: Cancer stem cells - new and potentially important targets for the therapy of oral squamous cell carcinoma. Oral Dis 12: 443-454, 2006.

21. Locke M, Heywood M, Fawell S and Mackenzie IC: Retention of intrinsic stem cell hierarchies in carcinoma-derived cell lines. Cancer Res 65: 8944-8950, 2005.

22. Izumiya M, Kabashima A, Higuchi $\mathrm{H}$, et al: Chemoresistance is associated with cancer stem cell-like properties and epithelial-to-mesenchymal transition in pancreatic cancer cells. Anticancer Res 32: 3847-3853, 2012.

23. Moon JH, Kwon S, Jun EK, et al: Nanog-induced dedifferentiation of p53-deficient mouse astrocytes into brain cancer stem-like cells. Biochem Biophys Res Commun 412: 175-181, 2011.

24. Chen SL, Iddings DM, Scheri RP and Bilchik AJ: Lymphatic mapping and sentinel node analysis: current concepts and applications. CA Cancer J Clin 56: 292-309, 2006.

25. Trivedi NP, Ravindran HK, Sundram S, et al: Pathologic evaluation of sentinel lymph nodes in oral squamous cell carcinoma. Head Neck 32: 1437-1443, 2010.

26. Kayahara H, Okuda M, Terakado N, Shintani S and Hamakawa H: Non-randomized clinical study comparing chemotherapy plus radiotherapy with radiotherapy alone in neoadjuvant therapy for oral cancer. Gan To Kagaku Ryoho 29: 911-916, 2002 (In Japanese). 